\title{
Weighing the impact of ICT on "modular" SMEs
}

\author{
Alessandro Marchetto, Mattia Monga, Andrea Trentini * \\ D.I.Co. - Università degli Studi di Milano \\ Via Comelico 39, 20135 Milano, Italy \\ (marchetto|monga|trentini)@dico.unimi.it
}

\begin{abstract}
We call "modular firm" a network of small interacting firms, exchanging artifacts (atoms) and information (bits) to accomplish a common goal. In this paper we propose a method to assess how effective is the information exchange among firm modular units. We propose to model a modular firm as a set of units that interact through well-defined channels: structural information together with type, format, and traffic shape are used to weigh the information exchange and the value associated to a given organizational setting.

Keywords: SME, delocalization, ICT
\end{abstract}

\section{Introduction}

As the complexity of business processes grows, modern firms tend to organize themselves in networks of task oriented units. The goal of managing complexity drives this trend, but a truly modular organizational setting- where units interact only through predefined transactions- enables parallel work and may improve the flexibility with respect to the uncertainty of the future [BC03]. Thus, we introduce the notion of a "modular firm" as a network of small interacting firms, exchanging artifacts and information to accomplish a common goal.

Artifacts (atoms) have to be physically transported (using some kind of vehicle) while information (bits) can obviously be digitally transmitted through information networks (e.g. telephone, Internet, etc.). Intuitively, the nature (its format and/or packaging) of what is exchanged has an impact on the value of the information exchanged. In this paper we focus on information bits and we claim that a critical factor that influences the value of communication is its format: a machine-parsable format makes information more useful in the production process. However, introducing a

* The authors gratefully acknowledge financial contributions from the FIRB project "International fragmentation of Italian firms. New organizational models and the role of information technologies", a research project funded by the Italian Ministry of Education, University and Research. parsable format may have associated costs, thus an analysis of information flows is needed in order to understand where formatting efforts are best placed.

In our model of a modular firm, units act as black boxes that produce and/or consume atoms and bits via predefined transactions. Transactions travel over channels between nodes. This view is very similar to object-oriented modeled systems where instances interact one another by sending messages. We would like to discuss about the value of this "message traffic" in a non software world.

The quality of data traffic is heavily affected by:

- the nature (type) of what is exchanged during a transaction, i.e., its intrinsic binding value;

- the format used to codify embedded information since some formats are better than others to exploit the knowledge in further steps of the business process;

- the shape of the exchanged traffic in terms of:

- channel features (e.g. speed, cost)

- frequency of exchange (number of transmissions per day/week/etc.)

Our goal is to design a method to evaluate the information transfer efficency of a modular firm through the definition of an abstract model that can be applied by gathering structural information on actual firms under analysis. Our model contains a "weight table" that can be used to measure the exchange value of any channel between firms, then, by summing up (average actually) every channel we obtain the information exchange value in the whole network.

Modularization is usually power, since a modular firm can be more flexible and adaptive to market evolution, labor costs fluctuations, etc. But modularization takes its toll, since any exchange of atoms or bits has nonzero costs. The costs of physical transportation depend on physical weight and distance, while the costs of information transfer are heavily influenced by the type of information representation, distance in terms of space and time can often be ignored. 
The idea is very simple:

- gather information about all the data flows in the network of firms

- apply weights (Section 3)

- compute network value (in terms of information exchange)

Our method can be used to evaluate a network and to identify the smallest (thus less expensive) set of changes (e.g. in terms of data formats) than can be applied to the network to increase its value.

Section 2 is a "related works" overview, section 3 describes our methodology proposal, section 4 applies the methodology to an example network.

\section{Related works}

In this section we give an overview about the state of the art in terms of firms modularization and ICT.

Several works study firms (or network of firms) modularization, architectures and design. [BC03] discusses the value of modularization aimed at making complexity manageable, enabling parallel work and accommodating future uncertainty. It presents the process performed to modularize a system and it identifies six basic modularization operators: substitution, splitting, augmentation, exclusion, inversion, and porting. The process focuses on the description of the general architecture and "decision rules" to govern it, the composition of basic modules and their correlated interfaces specifications. [M.02] details the state of the art about modularity in design, production and firms organization through a (critical) literature review. [Ben02] discusses the current interpretation of modularity in business and organization. Moreover, it analyzes modularity attributes as they emerge from analysis of several (manufacturing) case-studies. Another work [TVS04] focuses on the analysis of product and process modularization where product modularity is introduced as the practice of using standardized product modules so they can be easily reassembled/rearranged into different functional forms, or shared across different product lines. Instead, process modularity is introduced as the practice of standardizing manufacturing process modules so that they can be resequenced easily or new modules can be added quickly in response to changing product requirements. Other interesting works are focused on the modularization cost and exchanged information. [BC03] describes this cost as based on the kinds of modularity and dependencies among modules. Instead, [BC02a] defines the "net option value" of a particular module as influenced by: its "technical potential", the cost of mounting independent design experiments, and the "visibility" of the module in question. Using this value the authors suggest a mathematical approach to calculate the option value of a system (of modules). This value is used to define when investment in a new modular architecture are justified. [BC02b] focuses on the exchanged information on a firms network. It introduces three types of information: data (information about tasks and requirements), design (i.e., algorithms or processes that are solutions to problem posed by data) and tags (e.g., special information such as resources and "tasks" location in the firms network).

Furthermore, some works focus on the evaluation of design modularization. For example, [SGCH01], [CS06] and [Gin02] studies approaches and measures to analyze available modularizations (e.g., and to decide which is the best) and the complexity of their structures.

More strictly related with the goal of this paper, there are several works that analyze the relationships between firms modularization and ICT (e.g., the economical impact of ICT in firms business) and studies that classify ICT technologies and services (e.g., based on actors, technologies and use). [WGE05] studies networks firms when exchanged objects are ICT services, the author proposes a multi-layers approach to analyze networks that is focused on the businessICT alignment (i.e., the problem of matching ICT services with the requirements of the business). [BJ] studies how ICT influence a firm's boundary choices in terms of firm boundary decisions, organizational design and information systems. [McG04] studies how IT generate change within firms and how firms can use IT for strategic advantage. It focuses on the concepts of information, modularity organization and module connectivity. Morevoer, it introduces the "architecture potential" rate (based on IT and organizational modularity) to evaluate a given architecture. [Pil04] studies the impact of ICT in firms economical performance. It shows that ICT is having far reaching impacts on economic performance and the success of individual firms, in particular when it is combined with investment in skills, organisational change, innovation and new firm creation. [OEC04a] and [OEC04b] propose an interesting classification of ICT manufacturing (e.g., Office, accounting and computing machinery) and services (e.g., IT consulting). The former classifies ICT manufacturing through the following categories: telecommunications equipment, computer and related equipment, electronic components, audio and video equipment, other ICT goods. Thus, for every category correlated manufacturers are interviewed. Instead, the latter focuses its services classification on the following categories: IT technical consulting, IT design and development, Hosting and IT infrastructure provisioning, IT infrastructure and network management, IT technical support services, Information and document transformation, Internet access and backbone services, Published Software. [TŁCR02] provides a method for structuring the field of construction information technologies (e.g., for stucturing 
bibliographic and knowledge databases, research topics or projects). The built map is subdivided in core themes and support themes. The support themes address the research needs, transfer, deployment and impact of research. Core themes are related to processing activities and communication/coordination activities, they are subdivided in communication, processing and common infrastructures according to the human activities. Finally, communication is further split by the actors involved and processing is split according to information life-cycle.

Modularity in the firm structure may emerge to cope with the "natural" limit on the size of organizations. According to Coase [Coa37], when the cost of achieving a certain outcome in the world that requires human behavior through organizational means is lower than the cost of achieving that same result through implementation of a pure peer-to-peer price system, organizations will emerge to attain that result. An organization will cease to grow when (a) another organization can achieve the marginal result that they seek to obtain at lower cost; or (b) the price system can obtain that result at lower cost than can an organization.

\section{Methodology}

As defined above, a "modular firm" is a network of small firms, interacting one another by exchanging artifacts and information to accomplish a common goal. In Figure 1 the reader can find a UML[srw] (Unified Modeling Language) class diagram describing an abstraction of a network of firms. The three classes at the left hand side (Firm, DelocalizedUnit and Channel) describe the structural aspect of the network: the Firm is an aggregation of small units (DelocalizedUnit), these units are linked (many to many) through Channels. The right hand side of Figure 1 describes the available objects that can be exchanged among the units. At the top of hierarchy an abstract Exchangeable class that is subclassed into two main families: Artifact (atoms) and Information (bits). Without any loss of generality, in our proposal we focus only on Information exchange. The methodology we propose can in principle be extended to Artifacts by taking into account they too transfer information through their attributes. Instances of Information subclasses will be transferred through the network by using predefined Channels. Our goal is to assign value to those classes and then to frame the assigned values into a set of formulas to compute the overall network value. The set of classes should not be considered complete, instead domain experts may refine the model by adding/removing classes and by assigning values according to the context under examination.

The classes we identified (the list of subclasses of Information can be augmented at will) are the following:

Firm: the aggregator of the set of small units;
DelocalizedUnit: (or modular unit) the single unit participating in the set;

Channel: the link between a pair of units;

Exchangeable: (abstract class) represents anything that can be exchanged through the network;

Artifact: (abstract class) atoms, artifact phisycally transported through the network;

Information: (abstract class) bits, generic information transmitted through the network;

Catalog: a list/description of available products;

PriceRequest: a price (and availability info) request for a single lot (set of products);

Price: pricing information for a single lot;

Order: request for an actual lot of products;

StatusRequest: info request about the work-in-progress status;

Invoice: request for payment;

Payment: actual info about a payment (e.g., bank transfer number)

Confirmation: approval;

Memo: generic information;

Project: description of a product, such as a schema, design plan, etc.

\subsection{Weighing the exchange}

Our model takes into account a set of modular units connected by channels where data are exchanged. We assume (see Section 1) that the exchange weight is influenced by: the type of information, its format, and traffic shape. Let $\mathcal{C}$ the set of all channels $C$ and $\mathcal{I}$ the set of all exchanged items $I$. Moreover, we define:

- $V_{I}$, the intrinsic/content value of an item $I$ (range $0 . .1$ )

- $F_{I}$, the representation format value of an item $I$ (range $0 . .1)$

- $S_{C}$, the speed of channel $C$ (range $\left.0 . .1\right)$

- $K_{C}$, the cost of channel $C$ (range $0 . .1$ )

- $f_{(C, I)}$, the frequency of exchanging item $I$ on channel $C$ (range $0 . .1$ ) 

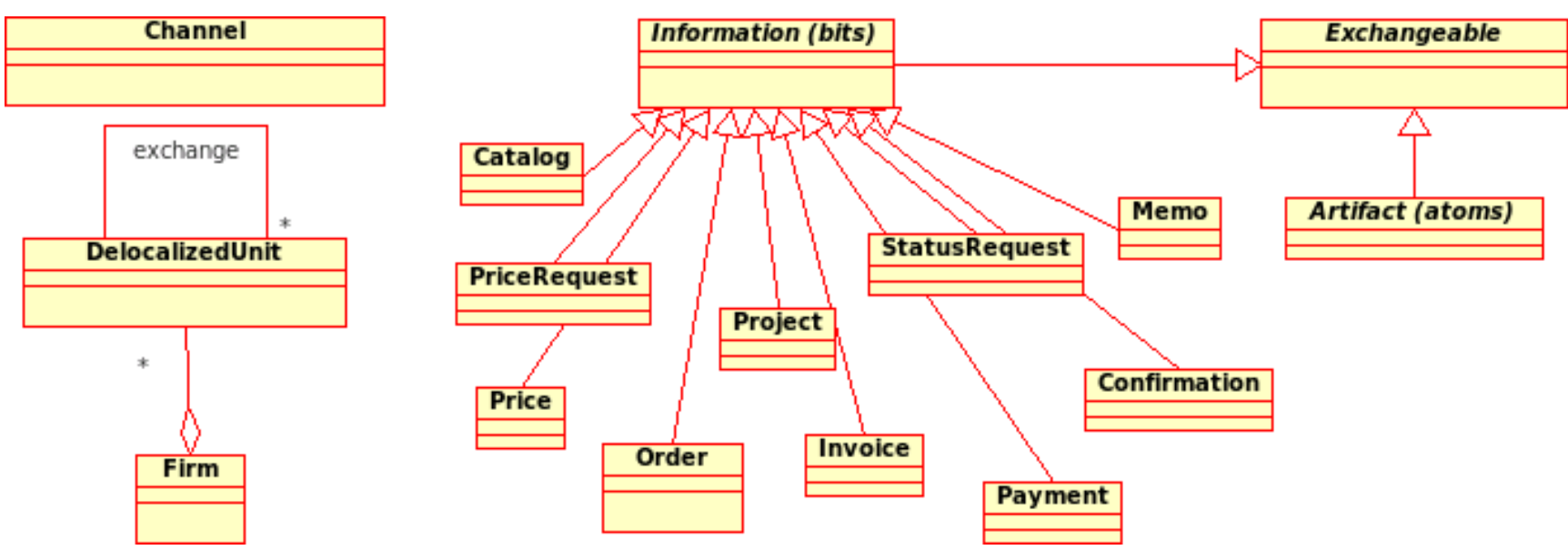

Figure 1. Model

Range values are not percentages, they are just values analytically set to rank a set of items.

Then, the efficiency $\eta_{C}$ of a single channel $C$ in $\mathcal{C}$ can be defined as:

$$
\eta_{C}=\left(S_{C} / K_{C}\right)
$$

Values for $S_{C}$ and $K_{C}$ need only to be comparable and can be relative, i.e., they can be pure numbers to weigh relative speeds and costs of channels in the network of firms.

Values for $V_{I}$ must be assigned contextually, i.e., the value of any Exchangeable instance will have to be negotiated with business domain experts, since the relative value of, say, Price information can be higher than Memo in a particular context.

The value exchanged on a channel, function of the value of transferred information, can be defined as:

$$
\text { Value }_{C}=\sum_{i \text { in } \mathcal{I}} f_{(i, C)} *\left(V_{i}+F_{i}\right) / 2
$$

(where $\left(V_{i}+F_{i}\right) / 2$ is the average between $V_{i}$ and $F_{i}$ )

Finally, the overall value of a channel can be defined as:

$$
\text { OverallValue }_{C}=\eta_{C} * V_{C}
$$

Then we can compute the average value (from the information exchange point of view) of the network of firms:

$$
\text { NetworkValue }=\left(\sum_{\text {in } \mathcal{C}} \text { OverallValue }_{c}\right) /|\mathcal{C}|
$$

\subsection{Format value}

Format value deserves a digression. Our role as ICT experts is to assign a relative value to formats used to repre- sent information, since the choice of format can heavily influence the value of a comunication, no matter the content. The value of data flow between unit pairs is greater if the transmission format is well known, standardized, machine readable, etc.

At the top of abstraction, only two macro categories are enough to distinguish formats: parsable and unparsable information. Parsable information can be automatically managed (read and understood) by a machine (i.e., a computer) without human intervention. Parsable information uses a format whose syntax is explicitly known to the receiver of the message. For example, an executable script to drive a production machine is "parsable", while any MSWord document is not ${ }^{1}$. Thus, parsable information has far more relative value than unparsable information.

A possible assignment for $F$ values is the following:

unparsable formats $(0-0.1)$

voice, either direct person to person or by phone ${ }^{2}(0-$ $0.05)$

unformatted text, e.g. fax or natural language email $(0.05-0.1)$

parsable formats $(0.1-1.0)$

formatted text with closed/proprietary format $(0.1$ $0.5)$

formatted text with open format $(0.5-1.0)$

\footnotetext{
${ }^{1}$ In a sense, if the receiver owns a copy of the MSWord application, she does know the syntax of the document. However, since this information is embedded in a closed source application, it is generally hard to exploit conveniently that knowledge.

${ }^{2}$ If the technology of voice recognition improved, this piece of information could be partially parsable and its value would increase
} 
The assignment above should be taken as a working proposal. However, it reflects our claim that parsable information is intrinsically more valuable than unparsable one. We believe an other important factor influencing our value assignment is the distinction between closed and open formats. While there is no unique definition of open format yet, we do adopt the Wikipedia definition [doof]: "An open format is a published specification for storing digital data, usually maintained by a non-proprietary standards organization, and free of legal restrictions on use."

We decided to give more value to open formats because they represent an important asset when used as communication facilities instead of closed/proprietary formats. In fact, open formats are:

- usually well documented

- freely usable

- community standards (de facto or de iure)

- often implemented by free/libre/opensource software ([weba], [webb])

- often "human readable" (i.e., editable even without the sw application originally used to create the information)

Instead closed formats are:

- usually poorly documented (or the documentation have to be purchased from a single vendor)

- not always freely usable (since sometimes the vendor requires that royalties are payed for adopting a proprietary format)

- community standards (usually de facto, such as MSOffice)

- rarely implemented by free/libre/opensource software (because of vendor charges to acquire rights to implement)

- rarely "human readable" (because the software vendor is not interested in revealing its "industrial secrets")

Given this comparison of features, since we are studying a network of interacting firms, we decided to assign higher values to means that facilitate interchange and substitution and avoid vendor lock-in.

The change of format in a firm its an expensive operation, since it must be implemented by a combination of the following:

switching software: building/acquiring new software, with the probable added training personnel costs

\begin{tabular}{|c|c|c|}
\hline Type & Format & Value \\
\hline \multicolumn{3}{|c|}{ Email attachment } \\
\hline text only & unpar. text & 0.05 \\
\hline XML & par. text & 1 \\
\hline specs (text) & unpar. text & 0.1 \\
\hline Excel & par. prop. & 0.4 \\
\hline CSV sheet & par. open & 1 \\
\hline graph (dot) & par. prop./open & 0.5 \\
\hline UML & par. open & 1 \\
\hline script (linux) & par. open & 0.6 \\
\hline PDF/PS & unpar. text & 0.06 \\
\hline \multicolumn{3}{|l|}{ project } \\
\hline graph & par. prop./open & 0.5 \\
\hline UML & par. open & 1 \\
\hline text only & unpar. text & 0.06 \\
\hline specs (text) & unpar. text & 0.1 \\
\hline XML & par. open & 1 \\
\hline
\end{tabular}

Table 1. Technological Possibilities

adding wrapper software: building/acquiring interfacing software, not always an easy task

Of course these costs must be weighed by CEOs and CIOs to decide where to optimize.

\section{Example}

In this section we detail an example of use in order to show how to apply our method. In particular, given a network of firms, we want to evaluate some possible scenario of "exchanged information". The sample-network of firms considered in this sample is composed of three nodes (firms here named orderl, prodl 1 and prod2) that exchange information (such as orders, tasks, etc.) as follows:

order 1 collects orders and controls the production process (e.g., orders to produce and distribute shoes)

prod1 produces a specific artifact (e.g., shoes production)

prod2 does a specific task (e.g., shoes distribution)

Now we need to know how nodes exchange information in the network and the kind of information. In our example, order 1 sends commitments to prod 1 and $\operatorname{prod} 2$, while there is no information exchanged between these last nodes. $\mathrm{Or}$ derl orders products to be built or tasks to be accomplished through email (with/without attachments) and project plans written in electronic documents and sent via Internet. In more detail, we suppose that orderl sends "textual" email to prodl for ordering (e.g., the order may contains information such as the kind of shoes, the quantity, the models, and so on). Instead, it sends email with "semantic" attachment (e.g., a MSword document) to prod2 to describe and explain the need to perform for the shoes distribution task. Moreover, orderl sends project plains described into electronic documents to both other nodes.

Table 1 shows a list of "Technological Possibilities" (i.e., format of email, attachments and/or electronic documents) 


\begin{tabular}{|c|c|c|}
\hline & order $1 \rightarrow$ Prod 1 & order $1 \rightarrow \operatorname{Prod} 2$ \\
\hline \multicolumn{3}{|c|}{ Scenario 1 } \\
\hline $\begin{array}{l}\text { email } \\
\text { project }\end{array}$ & $\begin{array}{l}\text { text only } \\
\text { graph }\end{array}$ & $\begin{array}{c}\text { graph (dot) } \\
\text { specs }\end{array}$ \\
\hline \multicolumn{3}{|c|}{ Scenario 2} \\
\hline email & XML & XML \\
\hline project & UML & UML \\
\hline \multicolumn{3}{|c|}{ Scenario 3} \\
\hline email & text only & Excel \\
\hline project & text only & specifiche \\
\hline \multicolumn{3}{|c|}{ Scenario 4} \\
\hline $\begin{array}{l}\text { email } \\
\text { project }\end{array}$ & $\begin{array}{l}\text { text only } \\
\text { text only }\end{array}$ & $\begin{array}{l}\text { CSV sheet } \\
\text { specs }\end{array}$ \\
\hline
\end{tabular}

Table 2. Scenarios

usable by orderl to send its (electronic) orders. In this table, for every listed format-type, we describe its F (representation format value) according to the criteria suggested in Section 3 (e.g., parsable/unparsable). Moreover, the table contains a column of numerical value for the $\mathrm{F}$ assigned based on the format type and the automation level of every possible technology. For example, a "text only" (e.g., a description of task required in natural language) email attachment is considered unparsable text and, according to the criteria described in Section 3.2, its value is 0.05 . Instead, an attachment written in MS-Excel format it is considered (automatically) parsable text and its format is closed/proprietary (i.e., we need to use MS-Excel software to read/write it) thus, its value is 0.4 .

To evaluate the information exchanged among firms of our network, table 2 describes four possible scenarios. In every scenario we describe the electronic format selected by orderl to send information to the other network nodes. For example, in Scenario 1 orderl sends textual mail (e.g., email with textual attachment that describes requirements) to ask commitments to prodl while, it uses graph format (e.g., dot) for sending information related to a required project. On the contrary, orderl uses graph format (e.g., email attached in dot-format) to ask a distribution-task to prod2. Moreover, it uses an electronic document (manually) written using pre-compiled modules to describe to $\operatorname{prod} 2 \mathrm{a}$ required project.

Summarizing, we have a sample network of firms composed of three nodes that exchange information through two channels (i.e., A:order $1 \rightarrow$ Prod 1 and B:order $1 \rightarrow \operatorname{Prod} 2$ ) and thus, considering the four possible scenarios described here, we may evaluate the exchanged information of our network through the following steps (see Section 3):

1. for every channel we calculate the channel efficency $\left(\eta_{c}\right)$;

2. for every channel we calculate the channel value $\left(\right.$ Value $\left._{c}\right)$;

3. for every channel we calculate the overall value of the channel (OverallValue $)$
4. then using the average of all the computed OverallValue $_{c}$ we evaluate the network of the firm (NetworkValue)

Using all defined scenarios, we apply these steps for all related networks. Before going on, we need to consider that we assumed some simplifications:

1. we set connections channel speed $\left(S_{c}\right)$ and cost $\left(K_{c}\right)$ to 1 , so we consider the same channel efficiency for both channels (i.e., always $\eta_{c}=1$ )

2. we analyze only one communication that traverse our channel and we consider information frequency always equal to 1 (i.e., always $f=1$ )

3. we set the intrinsic/content value of the exchanged information equal to 1 (i.e., always $V=1$ )

Using these hypotheses we may proceed with our (4) networks evaluation. Therefore, for every scenario we calculate $V_{c}$ as the sum of $1 *\left(1+F_{i}\right) / 2$ for every exchanged information, where $F_{i}$ is the value related to the current information format. E.g., for Scenario 1 and channel A (i.e., order $1 \rightarrow$ Prod 1 ), according to the meta-model described in Figure 1, we need to consider the information for order and project. Thus, for channel A the related representation format value is $F_{\text {email }}=0.525$ for email format (i.e., only text) for and $F_{\text {project }}=0.75$ for project format (i.e., graph dot). Then, we may calculate the channel value as the sum of the information value for order and project. Therefore, the value of the channel A (in Scenario 1) is 1.25. Through the same approach we calculate $V_{c}$ for every channel in every scenario. Table 3 summarizes this data.

We recall here that in our sample we consider $\eta_{c}=1$

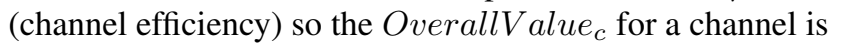
equal its channel value $\left(V_{c}\right)$ (as shown in Table 3 ). To conclude our computation we use the overall channel values to calculate the network values as the sum of the channel OverallValue $_{c}$ divided by the networks channel cardinality (i.e., 2 in our network). For example, the network value related to the Scenario 1 is 1.275 (see Table 3).

This basic example shows that the network value increases when using "valuable" formats, in a more complex (real world) example, when using actual values or $K_{c}$, $S_{c}, V_{c}$ and $f$, we could experience different results, with valuable-formats value possibly obfuscated by the network parameters (frequencies of transmissions, cost and speed of channels).

\section{Open issues}

Currently, our approach abstracts away the topology of the firm, i.e. how units are connected one with another. Even if we use the average to compute network values (i.e., 


\begin{tabular}{|c|c|c|c|c|c|}
\hline Scenario & $\mathrm{A}$ & $\mathrm{B}$ & $V_{c}(A)=$ OverallValue $_{c}(A)$ & $V_{c}(B)=$ OverallValue $_{c}(B)$ & $\mathrm{Nv}$ \\
\hline 1 & $\begin{array}{c}\text { order }=0.525 \\
\text { project=0.75 } \\
\text { order }=1\end{array}$ & $\begin{array}{c}\text { order }=0.75 \\
\text { project=0.55 } \\
\text { order }=1\end{array}$ & 1.25 & 1.3 & 1.275 \\
3 & $\begin{array}{c}\text { project=1 } \\
\text { order }=0.525 \\
\text { project }=0.53\end{array}$ & $\begin{array}{c}\text { project=1 } \\
\text { order=0.7 } \\
\text { project=0.55 }\end{array}$ & 2 & 2 & 2 \\
4 & $\begin{array}{c}\text { order }=0.525 \\
\text { order }=1\end{array}$ & 1.055 & 1.25 & 1.1525 \\
project $=0.53$ & 1.055 & 1.55 & 1.3025 \\
\hline
\end{tabular}

Table 3. values

adding nodes does not necessarily imply more value) we should factor in the added (hidden from the pure data flow analysis) management costs of a large network (e.g., integration and coordination costs[BC03]). In fact, our model does not value the dependencies introduced by every exchange.

In the following small network examples assume that the channels are identical in terms of value (set to 1) so that the three networks have the same overall value $(1 / 2)$, yet they have very different topologies and very different dependencies ( 1 step, $n$ step, ...) between them. How should we weigh these differencies?

chain, pipe $A \longrightarrow B \longrightarrow C$

with this dependency matrix:

$$
\begin{aligned}
& A \quad B \quad C \\
& \begin{array}{llll}
A & 0 & 0 & 0
\end{array} \\
& B \quad 1 \quad 0 \quad 0 \text { density: } 2 / 9 \\
& \begin{array}{llll}
C & 0 & 1 & 0
\end{array}
\end{aligned}
$$

( $\mathrm{C}$ depends from $\mathrm{A}$ in two steps, the transitive closure density is $3 / 9=1 / 3$ )<smiles>[3H][Al][30Br]</smiles>

with this dependency matrix:

$$
\begin{array}{lllll} 
& A & B & C & \\
A & 0 & 0 & 0 & \text { density: } 2 / 9 \\
B & 1 & 0 & 0 & \\
C & 1 & 0 & 0 &
\end{array}
$$

\section{loop $A \rightleftharpoons B$}

with this dependency matrix:

$$
\begin{array}{cccc} 
& A & B & \\
A & 0 & 1 & \text { density: } 2 / 4=1 / 2 \\
B & 1 & 0
\end{array}
$$

(A and $\mathrm{B}$ depend on each other)

We plan to integrate our method with measures of coupling (such as "fan-in" and "fan-out") and topology/graph measures (such as dimension, depth, number of nodes, number of archs, number of loops, dimension of loops, etc.).

\section{Conclusion}

This paper proposes a method to evaluate the overall value of data flow comunication in a network of small firms connected one another. The method assign values to content type, content format, frequency of transmission and channel features (in terms of speed and cost). Content type value is assigned by business domain experts, content format value is assigned by classifying tecnologies in variuos levels of "parsability" and "openness". The UML model can be augmented (i.e., by specializing the Information abstract class) to adapt the method to specific contexts.

The computation results can be used to identify hot spots (bottlenecks), to decide where (channels) and how (formats) to modify the data flow and, in general, to play what-if scenarios.

Our next steps will be:

- to take into account topological information (i.e., we only value the channel per se, we should also weigh in the dependency matrix representing the graph of firms);

- to refine our model (weights, ontology);

- to validate it using real world examples.

\section{Discussion}

Here follows a concise report of the discussion held during the Equity 2007 presentation session. Most comments are listed here but some of them have been assimilated throughout the text.

\subsection{Weight tables}

Is there a relationship (dependency) between the value of an item and the channel through which this item is sent? I.e., is the value of an item a combination of an intrinsic part and an extrinsic (contextual) one? Maybe. For example (this example was described by a conference participant, but after the discussion session), a product project is an inherently complicated information, is it possible to imagine that the value of this information is greater if it is carried (and explained) by hand? 
Maybe.

We may answer to this question with a suggestion: the human explanantion of the project is another type of information, it is another item with another intrinsic value. We may map this item by augmenting our model (class diagram) either by simply adding one class (such as HumanInfo) or by adding a combined class that embodies the project and the explanation (such as an hypothetical ProjectDocumentExplainedByHuman that specializes Project).

\subsection{Data gathering}

More than a single question was risen about the data gathering problem:

- How to collect data in general? This is hard! And Italy is a particularly harsh environment for this goal. We could tell horror stories about the "management" of structural information inside our firms... We know about many cases of non-existent/outdated written documentation about internal workflow, either for lazyness or to "protect self indispensability". Situations where reverse engineering is the only way to collect actual data about workflow and intra-firm connections. In general, a complete map of intra-firm dataflow can be built by interviewing managers (not only IT ones) and by reviewing workflow documentation (where available).

- Do you think that managers are able to give value to open formats? No... and yes. Again, here in Italy there is no great culture about the value of formats in general, let alone open ones. And our goal is somewhat double, in the sense that we are interested in the evaluation of a network of firms and we would also like to frame a discussion about open formats (and their greater value, in our opinion) inside the discussion about network value. Yet some managers (the savvy ones) do care about formats, but in general we have experienced more interest for the idea of standards (de facto, de iure) with less attention to their openness. To gather information about the acceptance of open formats (and open source) we have added some (direct and indirect) questions to an investigation carried out for this project among small firms. We asked if (and why) they are using opensource software, information about the structure of their firms, their expenses for ICT, the technologies they are using, etc. The investigation started at the end of february 2007, we are now collecting data back.

- Do you think that managers are able to give relative (in the sense of ranking) value to their data?
Again... Yes and no. A manager must know in detail the workflow of its firm and the relationships (connections) with other firms. Well... a good manager should know about this, the problem is to persuade him to share this knowledge, and knowledge is power...

\subsection{Tool}

After the first submission we begun the implementation of a tool to "play with numbers" (the examples presented here were built by hand and spreadsheet), alas we are currently at an early stage: the design of database structure. We plan to complete the tool as soon as we can and then feed data gathered from some firms we have contacted to ask them for their collaboration.

\section{References}

[BC02a] C.Y. Baldwin and K.B. Clark. The Option Value of Modularity in Design An Example from Design Rules, Volume 1: The Power of Modularity. May 2002.

[BC02b] C.Y. Baldwin and K.B. Clark. Where Do Transactions Come From? A Perspective from Engineering Design. In Saint-Gobain Centre for Economic Research 5th Conference, Paris, France, November 2002.

[BC03] C.Y. Baldwin and K.B. Clark. The Value, Costs and Organizational Consequences of Modularity. May 2003.

[Ben02] M. Benassi. Modularization in manufacturing. In European Academy of Management II, Stockholm, Sweden, May 2002.

[BJ] S. Billinger and M. Jacobides. Changing the Firm's Digital Backbone: How Information Technology shapes the Boundaries of a Firm.

[Coa37] Ronald Coase. The nature of the firm. Economica, 4(16):386-405, 1937.

[CS06] Y. Cai and K.J. Sullivan. Modularity in Design: Formal Modeling and Automated Analysis. In to submitt TOSEM, 2006.

[doof] Wikipedia definition of "open format". http://en.wikipedia.org/wiki/open_format.

[Gin02] F. Gino. Complexity measures in decomposable structures. In European Academy of Management II, Stockholm, Sweden, May 2002. 
[M.02] Calcagno. M. Dynamics of Modularity. A critical approach. In European Academy of Management II, Stockholm, Sweden, May 2002.

[McG04] J. McGill. IT and Strategic Industry Transformations. December 2004.

[OEC04a] OECD. A proposed classification of ICT goods. In Meeting of the Technical Subgroup of the Expert Group on International Economic and Social Classifications, New York, USA, October 2004.

[OEC04b] OECD. Classifying information and communications technology (ICT) services. In Meeting of the Technical Subgroup of the Expert Group on International Economic and Social Classifications, New York, USA, October 2004.

[Pil04] D. et al. Pilat. The Economic Impact of ICT. Measurement, Evidence and Implications. OECD Publications, USA, March 2004.

[SGCH01] K.J. Sullivan, W.G. Griswold, Y. Cai, and B. Hallen. The Structure and Value of Modularity in Software Design. In International Conference on Software Engineering and ACM SIGSOFT Symposium on the Foundations of Software Engineering, Vienna, AU, September 2001.

[srw] Object Management Group (UML standard representative) website. http://www.uml.org/.

[TVS04] Q. Tu, M.A. Vonderembse, and Bhanu T. S. Measuring Modularity-Based Manufacturing Practices and Their Impact on Mass Customization Capability: A Customer-Driven Perspective. Journal of Decision Sciences, 35(2):147, May 2004.

[TŁCR02] Turk, M. Łmid, T. Cerovsek, and J. Reflak. ICT Ontological Framework and Classification. Technical report, ICCI: Inter-Connecting Construction Industry Project, December 2002.

[weba] Free Software Foundation website. http://www.gnu.org.

[webb] Open Source Initiative website. http://www.opensource.org.

[WGE05] R. Wieringa, J. Gordijn, and van P. Eck. ValueBased Business-IT Alignment in Networked Constellations of Enterprises. In 1st International Workshop on Requirements Engineering for Business Need and IT Alignment (REBNITA 2005), 2005. 